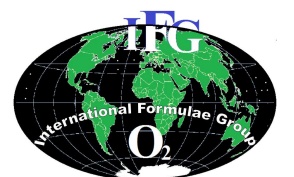

Review
Available online at http://ajol.info/index.php/ijbcs

Int. J. Biol. Chem. Sci. 8(6): 2740-2749, December 2014

ISSN 1997-342X (Online), ISSN 1991-8631 (Print)
International Journal

of Biological and

Chemical Sciences

\title{
Intérêt thérapeutique de la spiruline chez l'homme: revue général
}

\author{
Mamoudou BARRY ${ }^{1,4^{*}}$, Moussa OUEDRAOGO ${ }^{1,2,4}$, Seydou SOURABIE $^{5}$ et \\ Inocent Pierre GUISSOU ${ }^{2,3,4}$ \\ ${ }^{I}$ Centre Hospitalier Universitaire Pédiatrique Charles De Gaulle, Burkina Faso. \\ ${ }^{2}$ Unité de Formation et de Recherche en Sciences de la Santé (UFR/SDS), Burkina Faso. \\ ${ }^{3}$ Centre Hospitalier Universitaire Yalgado Ouedraogo, Burkina Faso. \\ ${ }^{4}$ Ecole Doctorale de Santé Université de Ouagadougou, Burkina Faso. \\ ${ }^{5}$ Institut de Recherche en Science de la Santé (IRSS), Burkina Faso. \\ *Auteur correspondant, Email : bmoudou@yahoo.fr; 01 BP 1198 Ouagadougou BF 01, Tél: 0022670409640
}

\section{RÉSUMÉ}

Le Spirulina platensis est une algue utilisée pour ses effets nutritionnel et thérapeutique. De nombreuses études ont montré son intérêt pour l'homme. Le présent travail résume les études cliniques effectuées avec la spiruline chez homme jusqu'en juin 2012. La méthode a consisté à l'interrogation des bases de données Pubmed, OMS, Medline base donné des Universités de Ouagadougou et de Grenoble, centre de pharmacovigilance de Grenoble et de Bordeaux. Seules les études effectuées chez l'homme avec la spiruline uniquement et publiées ont été retenues. Les critères de jugements ont porté sur les paramètres anthropométriques, biologiques, et des données du protocole. Ces études ont montré l'effet de la spiruline sur les maladies cardiovasculaires par la diminution des valeurs des paramètres lipidiques. La spiruline a eu des effets antidiabétiques par la diminution de l'hémoglobine glyquée et de la glycémie chez les patients diabétiques de type 2. Des effets immuno - modulateurs, anti-inflammatoires, anticancéreux, antianémiques, de la spiruline ont été prouvés. La récupération nutritionnelle de la spiruline a été prouvée dans six études dont trois chez les personnes vivant avec le VIH. Ces résultats montrent les multiples effets thérapeutiques de la spiruline mais doivent être confirmés par des échantillons de grandes tailles.

(C) 2014 International Formulae Group. All rights reserved.

Mots clés: Intérêt, thérapeutique, Spiruline, Homme.

\section{INTRODUCTION}

Les algues sont des espèces qui vivent toutes en milieu aquatique et sont presque toutes pourvues de chlorophylle. La spiruline est une algue bleue microscopique, pluricellulaire. Le genre Spirulina platensis ou Arthrospira, plus connu sous le nom d'algue Spiruline est une cyanobactérie de la classe des Cyanophyceae et de la famille des Oscillatoriaceae. Elle est riche en protéines, en lipides totaux, en glucides, en vitamines (liposolubles et hydrosolubles), en minéraux, en oligoéléments, et en pigments.

Plusieurs études ont démontré les possibilités de l'utiliser pour lutter contre la famine et la malnutrition dans le monde chez l'homme (Simporé et al., 2003, 2006; Teas et al., 2004; Sall et al., 2009;). En outre, elles ont montré l'intérêt thérapeutique de la spiruline (Bhavisha et al., 2010; Karkos et al., 2011) 
pour l'homme. Des études cliniques ont été menées chez l'homme pour plus de précisions sur l'implication de la spiruline dans plusieurs domaines thérapeutiques. Elles ont montré les effets thérapeutiques de la spiruline entre autre dans les maladies cardiovasculaires (Samuels et al., 2002) diabétiques (Parikh et al., 2001).

Le présent travail résume les résultats des ces études sur la spiruline chez l'homme. L'objectif de cette synthèse est de préciser l'intérêt de la spiruline, noté dans les études cliniques réalisées avec la spiruline chez l'homme.

L'étude a été menée sur des bases de données Pubmed, OMS, bases de données de la bibliothèque de médecine et de pharmacie de Grenoble, sur des bases des pharmacovigilances de Grenoble et de Bordeaux. Des sites internet spécifiques ont été consultés.

\section{Sélection des études}

Une première recherche a permis de retenir des articles sur la spiruline. Seuls ceux dont le produit de l'étude portait uniquement sur la spiruline chez l'homme et animal ont été retenus. Dans un deuxième temps, les études avec la spiruline chez l'homme et publiées ont été retenues (Figure 1).

\section{Les variables de l'étude}

Les paramètres biologiques ont été retenus pour apprécier l'effet thérapeutique de la spiruline. Les critères Z-score poids/âge (P/A), taille/âge (T/A), poids/taille (P/T) ont été utilisés pour l'appréciation de la récupération nutritionnelle des enfants.

\section{Période de l'étude}

Les études sur la spiruline chez l'homme jusqu'au 30 juin 2012, publiées et retrouvées ont été retenues pour l'étude.

\section{Effets de la spiruline sur des paramètres biochimiques chez l'homme}

Des études sur des volontaires ont montré que la spiruline contribue à augmenter le taux de cholestérol HDL et à diminuer les autres paramètres lipidiques (cholestérol total, cholestérol LDL, cholestérol VLDL, triglycérides) chez l'homme. Elle a diminué aussi le taux du glucose sanguin (la réduction de l'Hémoglobine glyquée (HbA1c) chez l'homme.

Ces modifications des paramètres lipidiques ont été observées avec des doses de spiruline allant de $1 \mathrm{~g} / \mathrm{j}$ à $8 \mathrm{~g} / \mathrm{j}$ pendant 6 semaines à 2 mois. En outre, une diminution des fréquences cardiaques et de production des apo-lipoprotéines ont été notées (Tableau 1et 2).

Rôle de la spiruline dans la régulation des éléments figurés du sang

La spiruline agit directement et indirectement sur la prolifération et la différenciation des cellules souches multipotentes de la moelle osseuse (Schwartz et al., 1987). Elle stimule l'hématopoï̀se, et surtout l'érythropoièse en induisant la libération de l'hormone l'érythropoïétine (EPO) (Mathew et al., 1995) (Figure 2).

Effets immuno-modulateur, antiinflammatoire, anticancéreux, hépatoprotecteur de la spiruline

La spiruline intervient au niveau du système immunitaire. Elle active les macrophages et les cellules NK. Elle induit la production d'anticorps, et active également les cellules T et B (Mathew et al., 1995). Elle a des effets immun modulateurs en augmentant la production des cytokines (interleukine-4 (IL-4), l'interféron- $\gamma$ (IFN- $\gamma$ ), l'interleukine2(IL-2) et de Natural killer (NK). Elle a des effets anti-inflammatoires, antianémiques (augmentation de l'hémoglobine), anticancéreux, hépato-protecteur (Tableau 3).

\section{Propriétés nutritionnelles de la spiruline chez l'homme}

Trois études cliniques chez l'homme ont montré l'intérêt de la spiruline dans la récupération nutritionnelle surtout chez les enfants (Branger et al., 2003; Simpore et al., 
2006; Sall et al., 2009). Les auteurs en s'appuyant sur les critères d'identification d'une malnutrition aiguë sévère des enfants âgés de 6 à 60 mois de l'OMS et de l'UNICEF, ont montré que la spiruline permet la récupération nutritionnelle de ces enfants. Les données anthropométriques ont montré une augmentation significative de la taille et $\mathrm{du}$ poids des enfants (Branger et al., 2003; Simpore et al., 2006; Sall et al., 2009).

\section{Effets de la spiruline chez les personnes infectées par le VIH}

Trois (3) études cliniques randomisées avec la spiruline ont été réalisées chez les personnes vivant avec le VIH. Elles ont montré les effets nutritionnels et immunostimulants de la spiruline (Simporé et al., 2006; Yamani et al., 2009; Marcel et al., 2011). La récupération nutritionnelle a été plus significative chez les enfants séronégatifs que chez les enfants séropositifs selon l'étude de Simpore (2006). Yamani (2009) et Marcel (2011), sans spécifier le statut sérologique, ont observé une récupération nutritionnelle moins significative avec la spiruline qu'avec le placebo. Simpore (2006) a observé une augmentation de l'hémoglobine chez les enfants infectés par le VIH. Yamani (2009) quant à lui, a observé une augmentation des protéines dans de sang.

Etudes sur la spiruline $(\mathrm{n}=139)$.
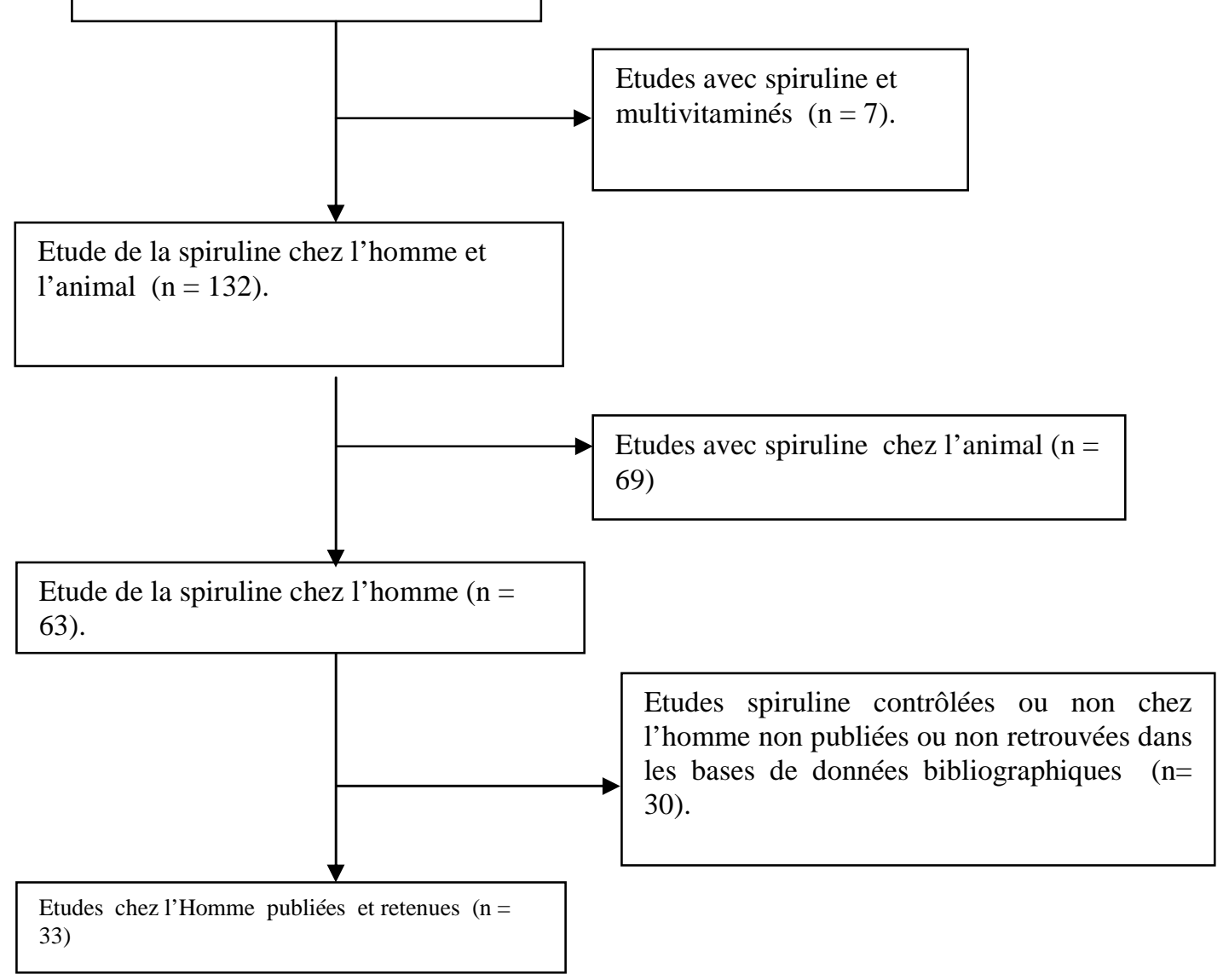

Figure 1: Sélection des articles de l'étude. 


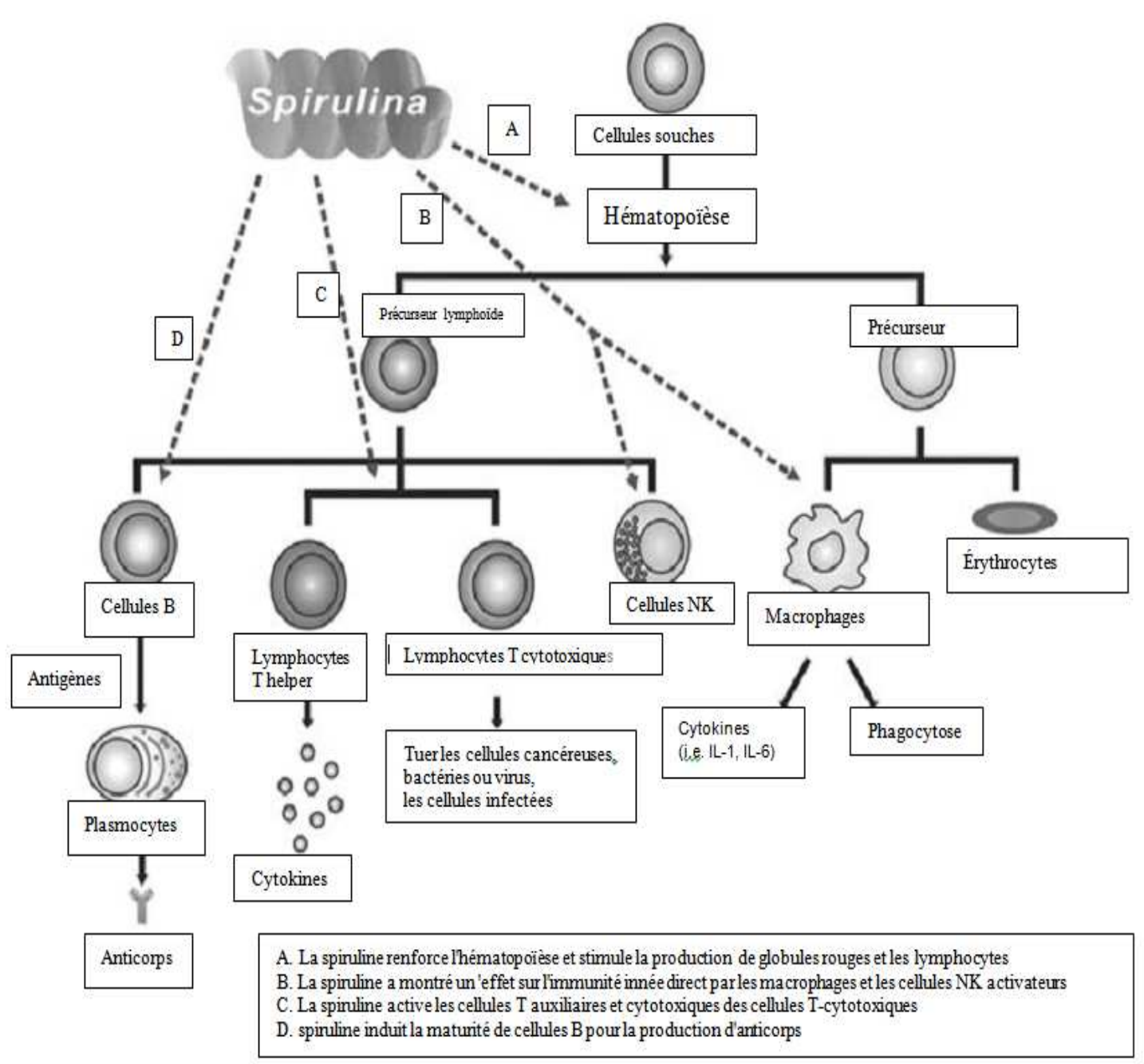

Figure 2: Rôle de la spiruline dans la production des globules rouges, des globules blancs et des anticorps (Schwartz et al., 1987). 
Tableau 1: Propriétés antilipémiantes de la spiruline chez l'homme.

\begin{tabular}{|c|c|c|c|c|c|}
\hline Sujets & $\mathbf{N}$ & Dose & Durée & Résultats & Références \\
\hline volontaires sains (Homme) & 30 & $4,2 \mathrm{~g} / \mathrm{j}$ & $\begin{array}{l}4 \text { ou } 8 \\
\mathrm{~S}\end{array}$ & $\begin{array}{l}\text { Réduction du cholestérol sérique total et de LDL et des triglycérides } \\
\text { Augmentation significative de HDL-cholestérol }\end{array}$ & Park et al.,2008 \\
\hline $\begin{array}{l}\text { Patients atteints de } \\
\text { cardiopathie ischémique }\end{array}$ & 30 & $\begin{array}{c}2 \text { à } \\
4 \mathrm{~g} / \mathrm{j}\end{array}$ & $3 \mathrm{M}$ & $\begin{array}{l}\text { Réduction significative de : cholestérol total, LDL, VLDL et de triglycérides } \\
\text { Augmentation significative de HDL } \\
\text { Réduction significative du poids corporel. }\end{array}$ & $\begin{array}{l}\text { Ramamoorthy et al. } \\
1996\end{array}$ \\
\hline $\begin{array}{l}\text { Patients atteints de syndrome } \\
\text { néphrotique }\end{array}$ & 23 & $1 \mathrm{~g} / \mathrm{j}$ & $2 \mathrm{M}$ & $\begin{array}{l}\text { Diminution significative : Cholestérol sérique total, cholestérol LDL, triglycérides } \\
\text { Diminution significative de ratios LDL / HDL et cholestérol total / HDL }\end{array}$ & Samuels et al., 2002 \\
\hline Volontaires en bonne santé & 36 & $4,5 \mathrm{~g} / \mathrm{j}$ & $6 \mathrm{~S}$ & $\begin{array}{l}\text { Réduction significative de taux de cholestérol plasmatique totale, LDL } \\
\text { Augmentation significative HDL } \\
\text { Diminution significative de la fréquence systolique et diastolique chez les hommes et les } \\
\text { femmes. }\end{array}$ & $\begin{array}{l}\text { Torres Duran et al., } \\
2007\end{array}$ \\
\hline Volontaires sains & 12 & $7,5 \mathrm{~g} / \mathrm{j}$ & $24 \mathrm{~S}$ & Diminution de : triglycérides, cholestérol total, LDL & Park et Kim, 2003 \\
\hline $\begin{array}{l}\text { Femmes âgées atteintes d' } \\
\text { hyperchole-stérolémie }\end{array}$ & 51 & $\begin{array}{c}7,5 \\
\mathrm{mg} / \mathrm{j}\end{array}$ & $8 \mathrm{~S}$ & $\begin{array}{l}\text { Réduction du cholestérol total, } \\
\text { Diminution de la production des apolipoprotéine B, IL-6 par les lymphocytes du sang } \\
\text { périphérique }\end{array}$ & Kim et al., 2005 \\
\hline Volontaires sains & 78 & $8 \mathrm{~g} / \mathrm{j}$ & $16 \mathrm{~S}$ & $\begin{array}{l}\text { Réduction significative de cholestérol total, } \\
\text { Pas de variations significatives des fractions LDL, HDL, de triglycérides (TG) }\end{array}$ & Park et al., 2008 \\
\hline Sujets de 18 à 65 ans & 36 & $4,5 \mathrm{~g} / \mathrm{j}$ & $6 \mathrm{~S}$ & $\begin{array}{l}\text { Augmentation non significative des ASAT et du glucose } \\
\text { Diminution significative TC et de HDL-C, LDL-C, TG. } \\
\text { Réduction de la tension artérielle systolique et diastolique }\end{array}$ & $\begin{array}{l}\text { Torres Duran et al., } \\
2007\end{array}$ \\
\hline
\end{tabular}

: : hich lensity lipopron : Diabète Non Insulino-Dépendant.

TG : triglycérides. 
M. BARRY et al. / Int. J. Biol. Chem. Sci. 8(6): 2740-2749, 2014

Tableau 2: Propriétés anticholestérolémiantes et hypoglycémiantes de la spiruline chez l'homme.

\begin{tabular}{|c|c|c|c|c|c|}
\hline Sujets & $\mathbf{N}$ & Dose & Durée & Résultats & Références \\
\hline Diabétiques de type 2 & 15 & $2 \mathrm{~g} / \mathrm{j}$ & $2 \mathrm{M}$ & $\begin{array}{l}\text { Réduction de : triglycérides, cholestérol total, LDL et VLDL, la glycémie, } \\
\text { protéines sériques Hb glyquée. }\end{array}$ & $\begin{array}{l}\text { Mani et al., } \\
2000\end{array}$ \\
\hline Diabétiques de type 2 & 25 & $2 \mathrm{~g} / \mathrm{j}$ & $2 \mathrm{M}$ & $\begin{array}{l}\text { Réduction : cholestérol total, LDL, triglycérides, glycémie } \\
\text { Augmentation : HDL. } \\
\text { Diminution significative des ratios cholestérol total / HDL et LDL / HDL } \\
\text { Baisse significative de l'apolipoprotéine B } \\
\text { Augmentation significative du niveau de l'apolipoprotéine A1 }\end{array}$ & $\begin{array}{l}\text { Parikh et al., } \\
2001\end{array}$ \\
\hline Volontaires sains âgés & 12 & $7,5 \mathrm{~g} / \mathrm{j}$ & $24 \mathrm{~S}$ & Diminution de triglycérides, cholestérol total, LDL & $\begin{array}{l}\text { Darcas et al., } \\
2004\end{array}$ \\
\hline Diabétiques de type 2 & 37 & $8 \mathrm{~g} / \mathrm{j}$ & $12 \mathrm{~S}$ & $\begin{array}{l}\text { Réduction de : LDL, triglycérides } \\
\text { Diminution de la pression artérielle et d'IL-6 }\end{array}$ & Lee et al., 2008 \\
\hline Diabétiques de type 2 & 60 & 1 ou $2 \mathrm{~g} / \mathrm{j}$ & $2 \mathrm{M}$ & $\begin{array}{l}\text { Diminution significative de : cholestérol sérique total, triglycérides, LDL, } \\
\text { VLDL dans les groupes de traitement de spiruline. } \\
\text { Diminution significative de la glycémie }\end{array}$ & $\begin{array}{l}\text { Kamalpreet et } \\
\text { al., } 2008\end{array}$ \\
\hline $\begin{array}{l}\text { Bénévoles masculins DNID agés } \\
\text { de } 45 \text { à } 60 \text { ans }\end{array}$ & 160 & $1 \mathrm{~g} / \mathrm{j}$ & $12 \mathrm{~S}$ & $\begin{array}{l}\text { Réduction significative de l'hémoglobine glycosylée et les niveaux du } \\
\text { profil lipidique du diabétique. } \\
\text { Augmentation du HDL }\end{array}$ & $\begin{array}{l}\text { Anitha et al., } \\
\qquad 2010\end{array}$ \\
\hline Volontaires & 30 & $4,2 \mathrm{~g} / \mathrm{jr}$ & $4 \mathrm{~S}$ & Réduction des cholestérols, TG et Cholestérol LDL et de glycémie & Nayaka , 1988 \\
\hline Sujets obèses & & $8,4 \mathrm{~g} / \mathrm{jr}$ & $4 \mathrm{~S}$ & $\begin{array}{l}\text { Effet hypocholestérolémiant; } \\
\text { perte de poids }\end{array}$ & Becker, 1986 \\
\hline
\end{tabular}
TG : triglycérides. 
M. BARRY et al. / Int. J. Biol. Chem. Sci. 8(6): 2740-2749, 2014

Tableau 31: Autres propriétés de la spiruline chez l'homme.

\begin{tabular}{|c|c|c|c|c|c|}
\hline Propriétés & $\mathbf{N}$ & Dose & Durée & Résultats & Références \\
\hline Immunomodulatrices & & & $12 \mathrm{~S}$ & $\begin{array}{l}\text { Mesure des cytokines (interleukine-4 (IL-4), l'interféron- } \gamma \text { (IFN- } \gamma \text { ) et } \\
\text { l'interleukine-2) } \\
\text { - Réduction significative de l'IL-4 (important dans la régulation de } \\
\text { l'immunoglobuline } \\
\text { (Ig) E-médiation allergie) } \\
\text { Production d'IgA (rôle central dans l'immunité mucosale) }\end{array}$ & $\begin{array}{l}\text { Mao et al., } 2005 \text {; Teas et } \\
\text { al., } 2004 \\
\text { Ishii et al., } 1999\end{array}$ \\
\hline & & & & Production d'IgA (rôle central dans l'immunité mucosale) & Ishii et al., 1999 \\
\hline & & & & Augmentation de la production d'IFN- $\gamma$ et NK & Hirahashi et al., 2002 \\
\hline Anti-allergiques & & & & $\begin{array}{l}\text { Amélioration des rhinites allergiques, d'écoulement nasal et éternuements du } \\
\text { groupe spiruline }(\mathrm{P}<0,001)\end{array}$ & Mao et al., 2005 \\
\hline Anticancereuses & 77 & & $12 \mathrm{M}$ & $\begin{array}{l}\text { - Régression tumorale après une application topique ou apport entéral d'extrait } \\
\text { de spiruline } \\
\text { - Régression complète de la leucoplasie ( } 45 \% \text { des patients de la cohorte) }\end{array}$ & Karkos et al., 2011 \\
\hline Antitoxiques & 41 & $250 \mathrm{mg} \times 2 / \mathrm{j}$ & $16 \mathrm{~S}$ & $\begin{array}{l}\text { - Comparaison des changements dans la peau (scores cliniques) et le contenu } \\
\text { d'arsenic dans l'urine et les cheveux } \\
\text { - Traitement de l'intoxication chronique à l'arsenic avec mélanose et de la } \\
\text { kératose. }\end{array}$ & Misbahuddin et al., 2006 \\
\hline Hématologiques & & & $12 \mathrm{~S}$ & $\begin{array}{l}\text { Augmentation : des valeurs de l'hémoglobine, du volume globulaire moyen, de } \\
\text { la concentration moyenne d'hémoglobine corpusculaire et des globules blancs }\end{array}$ & Deng et al., 2010 \\
\hline Hépatoprotectrices & 30 & $4,2 \mathrm{~g} / \mathrm{j}$ & $4 \mathrm{~S}$ & Réduction des cholestérols, TG et C- LDL et de Glycémie & Nayaka, 1988 \\
\hline
\end{tabular}


Synthèse

La spiruline a des propriétés nutritionnelles, antilipémiantes, et immunostimulantes. Elle pourrait être utilisée pour lutter contre la malnutrition surtout chez les personnes vivant avec le VIH. Les études sur les propriétés antilipémiantes de la spiruline (Park et al., 2008; Ramamoorthy et al., 1996; Samuel et al., 2002; Torres Duran et al., 2007) ont utilisé 1 à $8 \mathrm{~g}$ par jour de spiruline pendant deux semaine à 3 mois. Les études sur la récupération nutritionnelle des enfants (Branger et al., 2003; Simpore et al., 2006; Sall et al., 2009) ont utilisé 5 à $10 \mathrm{~g}$ de spiruline pendant 1 à 2 mois. Celle chez les personnes infectées par le VIH avec la spiruline, ont utilisé 5 à $19 \mathrm{~g}$ de spiruline pendant 2 à 6 mois. Ces données relèvent des insuffisances sur la posologie standard de poudre sèche de spiruline à être consommée par l'homme. Il serait nécessaire que des études soient réalisées pour lever cette inquiétude sur des éventuels surdosages qui donneraient des toxicités.

La spiruline a prouvé son intérêt thérapeutique (effets nutritionnels, hypoglycémiants, hypolipidémiants, immunostimulants, antitoxiques médicamenteux, anti-inflammatoires de la spiruline) chez l'homme. Elle pourrait être conseillée dans le cas de certaines pathologies telles que celles diabétiques, immunodépressives (comme le SIDA), hématologiques, inflammatoires cardiovasculaires, métaboliques. Ceci dans un cadre de surveillance biologique régulière.

La spiruline avec ses propriétés antioxydantes et d'antitoxiques, pourrait être conseillée dans des cas d'intoxication surtout avec les métaux et les médicaments pouvant générer des radicaux libres toxiques pour l'organisme humain. Cependant, celles qui ont évalué sa toxicité dans des études scientifiques rigoureuses telles que les essais cliniques sont rares voire inexistantes. C'est pourquoi des études de toxicité et de pharmacovigilance de la spiruline seraient nécessaires. L'évaluation des effets thérapeutiques cardiovasculaires et diabétiques des études cliniques avec la spiruline, ont été réalisées avec des échantillons de petite taille (maximum 78 personnes), sur une courte durée (maximum 3 mois). Des études cliniques randomisées contrôlées par des placebos à des posologies couramment utilisées chez l'homme adultes, seraient recommandées pour confirmer cellesci.

L'effet anti-diabète de la spiruline n'a été observé que chez les sujets de diabète de type 2 (insulinodépendant) et non chez le diabétiques de type1. Il devrait être confirmé chez des sujets non diabétiques sur une longue période. La spiruline pourrait être intégrée dans l'alimentation du diabétique sous des contrôles biologiques réguliers. Cependant, le rôle de la spiruline ou de ses constituants dans insulinosécrétion devrait être précisé par d'autres études.

Les effets immunostimulants et nutritionnels de la spiruline seraient bénéfiques aux patients infectés par le VIH qui connaissent une immunodépression et un déséquilibre nutritionnel. L'augmentation du taux d'hémoglobine par la spiruline promettrait son utilisation non seulement dans des pathologies qui induisent l'anémie telle que le paludisme dans nos pays mais aussi chez les personnes sous certains antirétroviraux (ARV) tels que la zidovudine (AZT) (OMS, 2010). L'utilisation de la spiruline chez les patients sous traitement ARV nécessiterait une étude de pharmacovigilance pour situer le rôle bénéfique ou nuisible de la spiruline avec ces ARV.

\section{Conclusion}

L'étude a permis de faire le point des études sur l'usage de la spiruline chez l'homme. Ce point de la littérature révèle des effets nutritionnels, antidiabétiques et antilipidiques, antianémique et autres effets thérapeutiques tels que ceux immunomodulateur, anti-inflammatoires, anticancéreux de la spiruline. Elle montre l'importance de la spiruline dans la lutte contre la malnutrition ainsi dans certaines pathologies. 
Des études avec de tailles élevées de population sont nécessaires pour reconfirmer les résultats.

\section{REMERCIEMENTS}

Nous remercions la Coopération Universitaire Franco-Burkinabé à travers son projet RESEAU qui a soutenu ce travail en nous octroyant une bourse. Nous remercions l'Université Joseph Fourier de Grenoble en particulier le personnel du service de la Pharmacie Clinique, du Centre de Pharmacovigilance et de la Bibliothèque.

\section{REFERENCES}

Anitha L, Chandralehha K. 2010. Effect of Supplementation of Spirulina on Blood glucose, Glycosylated Hemoglobin and Lipid Profile of Male Non-Insulin Dependent Diabetics. Asian J. Exp. Biol. Sci., 1(1):36-46.

Becker E. 1986. Clinical and biochemical evaluations of Spirulina with regard to its application in the treatment of obesity. Inst. Chem. Pfanz. Nutrition Reports International, 33(4): 565.

Bhavisha R, Parula P. 2010. Spirulina: Potential clinical therapeutic application. Journal of Pharmacy Research, 3(8): 1726-1732.

Branger B, Cadudal J, Delobel M, Ouoba H, Yameogo P, Ouedraogo D, 2003. Spirulina as a food supplement in case of infant malnutrition in Burkina-Faso. Arch Pediatr, 10(5):424-431.

Darcas C. 2004. Spiruline and malnutrition. Arch Pédiatrie Organe Off Sociéte Fr Pédiatrie, 11(5): 466-467.

Deng R, Chow T. 2010. Hypolipidemic, antioxidant, and anti-inflammatory activities of microalgae Spirulina. Cardiovasc Ther., 28(4): e33-45.

Hirahashi M, Matsumoto K, Hazeki Y, Saeki M, Seya T. 2002. Activation of the human innate immune system bySpirulina: augmentation of interferon production and NK cytotoxicity by oral administration of hot water extract of Spirulina platensis. International Immunopharmacology, 2(4): 423-434.
Ishii KTK, Okuwaki Y, Hayashi O. 1999. Influence of dietary Spirulina platensis on IgA level in human saliva. Journal of Kagawa Nutrition University, 30: 27-33.

Kamalpreet K, Rajbir S, Kiran G. 2008. Effect of supplementation of Spirulina on blood glucose and lipid profile of the noninsulin dependent diabetic male subjects. J. Dairying, Foods Home Sci., 27: 3-4.

Karkos P, Leong S, Karkos C, Sivaji N, Assimakopoulos D. 2011. Spirulina in clinical practice: evidence-based human applications. Evid-Based Complement Altern Med ECAM., 2011: 53103-53107.

Kim M, Kim WY. 2005. The change of lipid metabolism and immune function caused by antioxidant material in the hypercholesterolemin elderly women in Korea. Korean J. Nutr., 38: 67-75.

Lee EH, Park J, Choi Y, Huh K, Kim W. 2008. A randomized study to establish the effects of Spirulina in type 2 diabetes mellitus patients. Nutr Res Pract., 2(4): 295-300.

Mani I. 1998. Effect of wheat bran supplementation on serum glycosylated protein and serum lipid profile in maturity onset diabetic patients. Plant Fds Hum Nutr, 37: 184.

Mani U, Desai S, Iyer U. 2000. Studies on the long-term effect of Spirulina supplementation on serum lipid profile and glycated proteins in NIDDM patients. J. Nutraceut., 2(3): 25- 32.

Mao TK, Van de Water J, Gershwin ME. 2005. Effects of a Spirulina-based dietary supplement on cytokine production from allergic rhinitis patients. J Med Food., 8(1): 27-30.

Marcel A, Ekali LG, Eugene S, Arnold OE, Sandrine ED, von der Weid D. 2011. The Effect of Spirulina platensis versus Soybean on Insulin Resistance in HIVInfected Patients: A Randomized Pilot Study. Nutrients. 3(7): 712-724.

Mathew B, Sankaranarayanan R, Nair PP, Varghese C, Somanathan T, Amma BP. 1995. Evaluation of chemoprevention of 
oral cancer with Spirulina fusiformis. Nutr Cancer, 24(2):197-202.

Misbahuddin M, Islam AZMM, Khandker S, Ifthaker-Al-Mahmud, Islam N, Anjumanara. 2006. Efficacy of Spirulina extract plus zinc in patients of chronic arsenic poisoning: a randomized placebocontrolled study. Clin Toxicol (Phila)., 44(2): 135-141.

Nayaka.1988. Cholesterol lowering effect of Spirulina. Nutr. Rep. Int., 37: 1329-1337.

OMS. 2010. traitement antirétroviral de l'infection à VIH chez l'adulte et l'adolescent, recommandations pour une approche de santé publique.

Parikh P, Mani U, Iyer U. 2001. Role of Spirulina in the Control of Glycemia and Lipidemia in Type 2 Diabetes Mellitus. $J$ Med Food, 4(4): 193-199.

Park HJ, Lee YJ, Ryu HK, Kim MH, Chung HW, Kim WY. 2008. A randomized double-blind, placebo-controlled study to establish the effects of Spirulina in elderly Koreans. Ann. Nutr. Metab., 52(4): 322-328.

Park JY, Kim WY. 2003. The effect of Spirulina on lipid metabolism, antioxidant capacity and immune function in Korean elderly. Korean J Nutr., 36: 287-297.

Sall M, Dankoko B, Badiane M. 2009. Results of a nutritional rehabilitation with Spirulina in Dakar. Médecine d'Afrique Noire, 46(3): 143-146.

Samuels R, Mani UV, Iyer UM, Nayak US. 2002. Hypocholesterolemic effect of Spirulina in patients with hyperlipidemic nephrotic syndrome. J. Med. Food. 5(2): 91-96.

Schwartz . 1987. Regression of experimental hamster cancer by beta carotene and algae extracts. Journal of Oral and Maxillofacial Surgery, 45(6): 510-515.

Selmi C, Leung PSC, Fischer L, German B, Yang C, Kenny TP. 2011. The effects of Spirulina on anemia and immune function in senior citizens. Cell. Mol. Immunol., 8(3): 248-254.

Shklar G, Schwartz J. 1988.Tumor necrosis factor in experimental cancer regression with alphatocopherol, beta-carotene, canthaxanthin and algae extract. Eur $J$ Cancer Clin. Oncol., 24(5): 839-850.

Simpore J, Kabore F, Zongo F, Dansou D, Bere A, Pignatelli S. 2006. Nutrition rehabilitation of undernourished children utilizing Spirulina and Misola. Nutr J. 5:3.

Simpore J, Zongo F, Kabore F, Dansou D, Bere A, Nikiema J B. 2005. Nutrition rehabilitation of HIV-infected and HIVnegative undernourished children utilizing Spirulina. Ann. Nutr. Metab. 49(6): 373-380.

Teas J, Hebert JR, Fitton JH, Zimba PV. 2004. Algae a poor man's HAART? Med. Hypotheses, 62(4): 507-510.

Torres-Duran PV, Ferreira-Hermosillo A, Juarez-Oropeza 2007. Antihyperlipemic and antihypertensive effects of Spirulina maxima in an open sample of Mexican population: a preliminary report. Lipids Health Dis., 6: 33.

Yamani E, Kaba-Mebri J, Mouala C, Gresenguet G, Rey JL. 2009. Use of Spirulina supplement for nutritional management of HIV-infected patients: study in Bangui, Central African Republic. Med Trop., 69(1):66-70. 